\title{
Study on Asymmetric Two-Lane Traffic Model Based on Cellular Automata ${ }^{\star}$
}

\author{
Xianchuang $\mathrm{Su}^{1}$, Xiaogang $\mathrm{Jin}^{2, \star \star}$, Yong $\mathrm{Min}^{2}$, and Bo Peng ${ }^{2}$ \\ 1 College of Software Engineering, Zhejiang university, Hangzhou 310027, China \\ 2 AI Institute, College of Computer Science, Zhejiang university, \\ Hangzhou 310027, China \\ xiaogangj@cise.zju.edu.cn
}

\begin{abstract}
With the consideration of driver's velocity preference, we redefine the velocity updating rules of NaSch model and extend them to an asymmetric two-lane cellular automaton model with a recently proposed lane changing rule set. The analysis focuses on the reproduction of empirically observed results and the relations between empirical results and vehicle plugs. Vehicle plug is a structured vehicle set which slows down the traffic flow and it is also a bridge from microscopic to macroscopic level. Simulation results show that encouraging lane changes have little effect on decomposing vehicle plugs. In order to decrease plugs we should keep slow vehicles out of expressway or make a flexible overtaking ban of slow vehicle according to practical situations.
\end{abstract}

\section{Introduction}

In recent years, more and more cellular automata $(\mathrm{CA})$ models of traffic flow have been proposed. CA model formulate simple rules which mimic the behavior of the drivers as simply as possible, yield reasonable results compared with empirical findings and can be easily modified to study various instances. The basic model has been introduced by Nagel and Schreckenberg(NaSch model) [NS]. And a more sophisticated CA model [KSS1] has been developed which is capable of reproducing all of the empirically observed traffic states in single-lane traffic, i.e. free flow, wide moving jams and especially synchronized traffic.

In an asymmetric two-lane traffic only a few empirical results exist which help to specify lane changing rule[GY][HL]. Lane change frequency should increase with vehicle density, shows a maximum in the vicinity of the flow maximum and then decreases with increasing density. And a special feature of a highway with a right-lane preference is the empirically observed lane usage inversion. There are more vehicles distributed on the left than on the right lane, and the flow is larger for the left than for the right lane while vehicle density is over the vicinity

\footnotetext{
* Supported by Natural Science Foundation of China (NSFC) grant 60103015 and The Project-sponsored by SRF for ROCS, SEM.

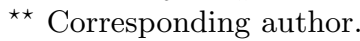


of the flow maximum. In [KSS2][KSS3][KSS4][RN] the two-lane extensions of NaSch model reproduce the density dependence of lane change frequency and the lane usage inversion quite well.

While the NaSch model only ensures the avoidance of crashes and every driver is only confined with a maximum velocity. For the desire of the drivers for smooth driving, we introduce ideal velocity with the consideration of individual velocity preference into NaSch model. Each driver takes speed at the vicinity of individual ideal velocity and moves on with the anticipation. Also the lane changing rules in [KSS3] could be mended by anticipation method which is capable of improving the usage of two-lane highway space and making the lane change process more neatly.

In the next section a modified asymmetric two-lane model is proposed not only for reproducing the empirical results on macroscopic level, i.e. density dependence of lane change frequency and the lane usage inversion, but also for letting the drivers in model have more human natures by reconstructing the local rule on microscopic level. In section 3 we introduce $U(t)$ which represent the average unhappiness of all drivers and show that by encouraging lane changing, $U(t)$ could stay at a low degree and the flow of traffic goes up linearly with the increasing density when the traffic is in free flow state. The influence of slow vehicle such as truck is remarkable in inhomogeneous asymmetric two-lane highway. Without the overtaking ban on truck, the flow would go down steeply with the increasing rate of trucks in all vehicles at the early stage and retain at a low level afterwards. The negative impact of truck rate on flow is vanished with the increasing vehicle density. We catch different segments of the highway and find that vehicle plug plays a main role in the negative impact. Once a vehicle plug is formed by a pair of trucks which occupy both two-lanes at short distance, the vehicles behind the plug are difficult to get through it. The relations between vehicle density, rate of truck and the formation of plug are discussed in detail. The plug is easy to form and spread all over but the measures that can dissipate it are difficult to find. Here we make some tries that may be helpful. In the last section a short summary and a discussion are followed.

\section{$2 \quad$ Models}

In this inhomogeneous asymmetric two-lane traffic model, a system update is performed in two sub-steps. In the first step the vehicles change lanes according to the lane changing rules and do not move. In the second step, the cars move according to the calculated velocity. Both sub-steps are performed in parallel for all vehicles. Before we present the lane changing rules, we briefly define the single-lane motion rules. For the sake of completeness we briefly recall the definition of the NaSch model. The NaSch model is a discrete model for traffic flow. The road is divided into cells which can be either empty or occupied by a car with a velocity $v=0,1, \cdots, v_{\max }$. The vehicles move from left to right on single lane with periodic boundary conditions and the system update is performed in parallel for all vehicles according to the following rules (see table 1 for a summary of the parameters and variables of models): 
Table 1. Summary of the variables and parameters used in the model definition

\begin{tabular}{|ll|ll}
\hline \multicolumn{2}{l||}{ Variable Parameter } & \multicolumn{2}{l}{ Variable Parameter } \\
\hline$x$ & Position & $R_{s}$ & Rate of slow vehicle \\
\hline$v$ & Velocity & $\rho$ & Vehicle density \\
\hline$v_{a}$ & Anticipation velocity & $v_{\max }$ & Maximum velocity \\
\hline$d$ & Distance headway & $d_{s}$ & Safe gap \\
\hline$d_{e}$ & Effective distance headway & $v_{i}$ & Ideal velocity \\
\hline$d_{o f e}$ & Effective distance headway other lane & $P_{b}$ & Deceleration probability \\
\hline$d_{o s e}$ & Effective distance behind other lane & $P_{a}$ & Acceleration probability \\
\hline
\end{tabular}

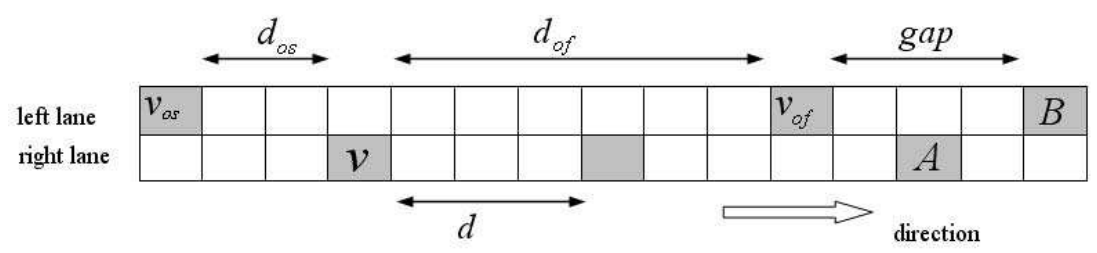

Fig. 1. Sketch of a road segment. Here take right lane as main lane and left lane as overtake lane. The hatched cells are occupied by vehicles

(1) Acceleration and deceleration:

$$
v(n, t+1)= \begin{cases}\min \left(v(n, t)+1, v_{\max }\right), & v(n, t)<d \\ d, & v(n, t) \geq d\end{cases}
$$

(2) Decelerated by noise: if $\operatorname{rand}()<P_{b}$ then $v(n, t+1)=\max (v(n, t+1)-1,0)$ (3) Motion: $v(n, t+1)=x(n, t)+v(n, t+1)$

where $v(n, t)$ and $x(n, t)$ denotes the velocity and the position of number $n$-th vehicle at system time $t$, respectively. With the deceleration probability $P_{b}$, various motions of drivers could be described in a random way. For the desire of the drivers for smooth and comfortable driving, Schneider et al[SE] and Knospe et al[KSS3] proposed a more realistic model based on NaSch model by introducing the effective distance headway $d_{e}$ :

$$
\left\{\begin{array}{l}
d_{e}(n, t)=d(n, t)+\max \left(v_{a}(n, t)-d_{s}, 0\right) \\
v_{a}(n, t)=\min (d(n+1, t), v(n+1, t))
\end{array}\right.
$$

where vehicle $n+1$ denotes the leading vehicle of $n$. The velocity of leading vehicle is anticipated and $d_{e}(n, t)$ allows for smaller gaps, larger velocity and higher lane space usage.

In real world, each driver has one's velocity preference. Here, we introduce the ideal velocity $v_{i}\left(v_{i}<v_{\max }\right)$ for each driver and the rules are designed to let the velocity varying around $v_{i}$. The status of the brake light $B$ is assigned to 1 when a deceleration occurred, otherwise $B$ is assigned to 0 . 
The update rules for motion are then as follows, which should be done step by step:

(1) Acceleration and deceleration: if $d_{e}(n, t)>v_{i}(n)>v(n, t)$ then $v(n, t+1)=$ $v(n, t)+1$; if $\left(d_{e}(n, t)>v_{i}(n)\right.$ and $v(n, t) \geq v_{i}(n)$ and $\left.\operatorname{rand}()<P_{a}\right)$ or $d_{e}(n, t) \leq$ $v_{i}(n)$ then $v(n, t+1)=\min \left(v(n, t)+1, d_{e}(n, t)\right)$; otherwise $v(n, t+1)=v(n, t)$;

(2) Decelerated by noise: if $\operatorname{rand}()<P_{b}$ then $v(n, t+1)=\max (v(n, t)-1,0)$;

(3) Upper limit: $v(n, t+1)=\min \left(v(n, t+1), v_{\max }\right)$;

(4) Brake light: $B(n, t+1)=\left\{\begin{array}{l}1, v(n, t+1)<v(n, t) \\ 0, v(n, t+1) \geq v(n, t)\end{array}\right.$

(5) Motion: $x(n, t+1)=x(n, t)+v(n, t+1)$.

The acceleration and deceleration rules of step one is designed for keeping the drivers driving at the vicinity of $v_{i}$ in free flow state, and the changing of velocity could be smoother. While in the state of synchronized traffic or wide moving jams, the velocities are constrained by the vehicle density. In order to extend the single-lane model to an asymmetric two-lane model, we should introduce lane changing rules which agree with the two mechanisms used in real life. The one is right-lane preference that the driver should use the right lane as often as possible. The other one is the right-lane overtaking ban. In [KSS3] a sophisticated lane changing rule set has been proposed, which agrees with the two mechanisms and is capable of reproducing the density dependence of lane change frequency and the lane usage inversion. In order to keep the model simply we restrict the lane interaction to vehicles which have to brake $(B=1)$ in the next time step due to an insufficient gap in front. The lane changing rules are as follows (see table 1 and figure 1 for the parameters and variables of rules):

(1) From right to left lane:

(i) Incentive criterion: $B=0$ and $v>d_{e}$

(ii) Safety criterion: $d_{o f e}>v$ and $d_{o s e}>v_{o s}$

(2) From left to right lane:

(i) Incentive criterion: $\left(B=0\right.$ and $\frac{d_{o f e}}{v}>2.0$ and $\left.\left(\frac{d_{e}}{v}>4.0\right)\right)$ or $\left(v>d_{e}\right)$;

(ii) Safety criterion: $d_{o f e}>v$ and $d_{o s e}>v_{o s}$ where $d_{\text {ofe }}$ denotes the effective gap to the leading vehicle on the destination lane, $d_{\text {ose }}$ denotes the effective gap to the succeeding vehicle on the destination lane, and they are defined as follows:

$$
\left\{\begin{array}{l}
d_{o f e}=d_{o f}+\max \left(\min \left(g a p, v_{o f}\right)-d_{s}, 0\right) \\
d_{o s e}=d_{o s}+\max \left(\min \left(d_{o f}, v\right)-d_{s}, 0\right)
\end{array}\right.
$$

It is obviously that the difference between two incentive criterions agrees with the right-lane preference mechanism. Since the velocity of the vehicle is taken into account in the incentive criterion that from left to right, slow vehicles are allowed to change lane even at small distances. 


\section{$3 \quad$ Results and Discussions}

The numerical simulations are started with randomly generated initial configurations, and evolve to steady terminal states over a long time. On a $2 \times 20000$ lattice as figure 1, trucks and cars are randomly distributed. We distribute the $v_{i}$ of trucks according to a Gaussian profile with different variances and a mean $\bar{v}_{i}=5$. While the $\bar{v}_{i}$ of cars is set to 8 , and $v_{\max }=12$. The sum of left and right lane usages is 2 . In order to describe the emotions of drivers, we introduce $U(t)$ which reflects the unhappiness of all drivers who are not at their ideal velocities at system time $t$ :

$$
U(t)=\frac{1}{n} \sum_{j=1}^{n} \frac{\left|v(j, t)-v_{i}(j)\right|}{v_{i}(j)}
$$

In figure 2 the variables of traffic flow varying with the increasing vehicle density are depicted (the units only have a relative meaning) and agree with the empirically observed results well. With the increasing density at early stage, traffic flow goes up linearly, mean velocity keeps on a high level and unhappiness stays at a low level while the lane change frequency goes up steeply. And we

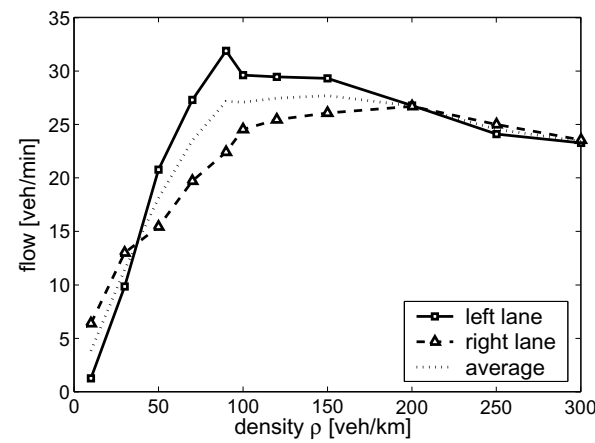

(a)

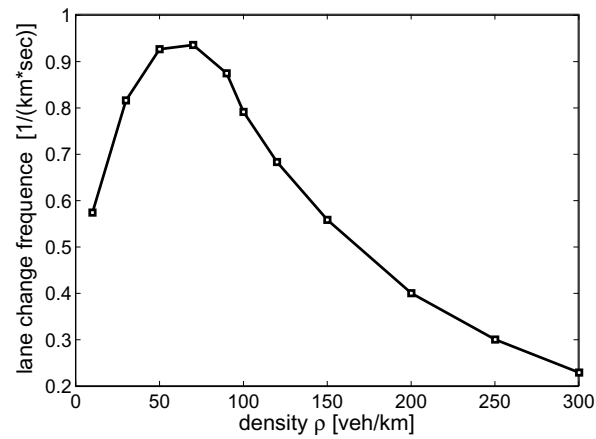

(c)

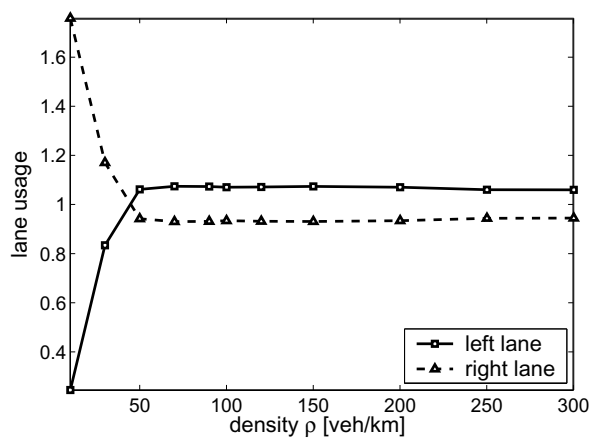

(b)

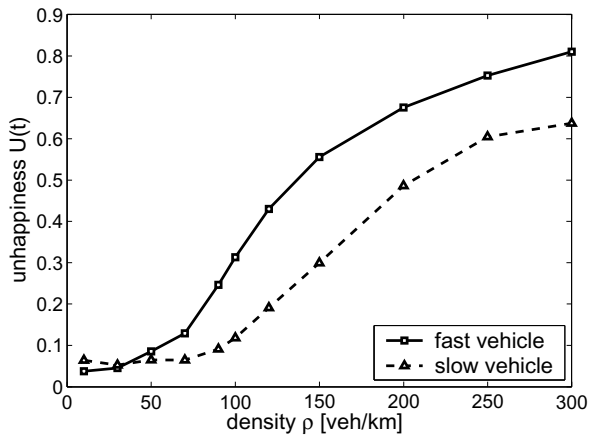

(d)

Fig. 2. Fundamental diagrams of asymmetric two-lane model with $10 \%$ slow vehicles.

The slow vehicles are not allowed to change to left lane 
can see that the active lane changes provide more chances for driver to drive at a high and satisfying velocity at free flow phase. Meanwhile, most of the vehicles are distributed on right lane for the right-lane preference. However, the left lane usage will exceed the right lane and then achieve its maximum in the vicinity of flow maximum, and the flow of left lane is larger than the right at the same time. A simple explanation for these is that the trucks or some slow cars dominate the right lane and the cars would like to take the left lane for more satisfying velocities. There are many factors in producing these phenomena, and a detail analysis is presented in [KSS3]. With the increasing density, the velocity predominance of cars against trucks is decreased, and the free flow phase turns into synchronized flow phase. In this stage the mean velocity of all drivers is determined only by density but not at the wills of drivers.

In real world, the entry of slow vehicle into highway is forbidden or the overtaking of slow vehicle is banned. Here the numerical simulations below will show us how the performances will be if we did not follow these instructions.

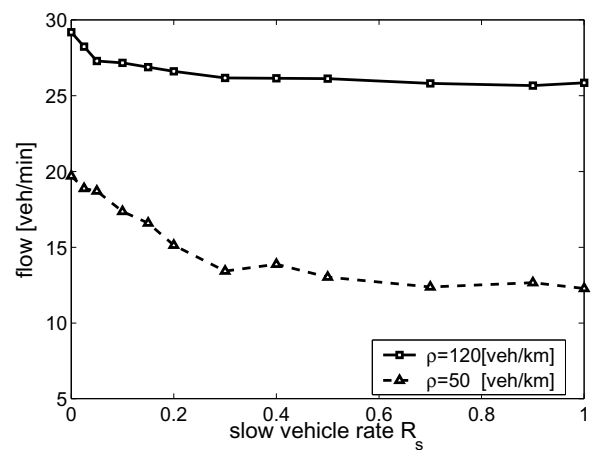

Fig. 3. Flow statistics of asymmetric tow-lane model under two different flow densities. Note that the trucks are allowed to change to the left lane

In figure 3 , when $\rho=50$ and the rate of trucks $R_{s} \in[0,0.27)$, flow goes down linearly and steeply. Meanwhile, the mean velocity of all drivers also has the same variation, and the lane change frequency goes up steeply with the increasing $R_{s}$ at this stage. However, when $\rho=50$ and $R_{s} \in[0.27,1]$, flow maintains at a low level, and so does the mean velocity. The lane change frequency decreases at the same time. All these phenomena are related to the slow vehicle plugs. In figure 1, we can see how the slow vehicle plugs are formed. Suppose A and B are all trucks, in the vehicle structure of figure 1 , the succeeding vehicles of $\mathrm{A}$ and $B$ will have few chances to go through the vehicle structure. And then more vehicles will be formed as slow vehicles at a segment with local high density which is called a vehicle plug. More and more vehicle plugs will be formed with the increasing $R_{s} \in[0,0.27)$ until they have been distributed everywhere. We tried to decompose the vehicle plugs by encouraging lane changing, i.e. tuning the numerical parameters in incentive criterion of lane changing rule that from 
left to right lane. But the result was even worse, since the flow enhanced only a little while the lane changing frequency increased doubly which would cause more crashes and make drivers tired and depressed. Therefore, it is important to keep slow vehicles out of expressway under these circumstances.

In figure 3 , when $\rho=120$, flow declines linearly but more steeply than the flow with $\rho=50$ and then it reaches a stationary value over a short range of $R_{s}\left(R_{s} \in[0,0.05)\right)$. Due to the vehicle plugs are easy to be formed than to be decomposed under higher density (the balance between plug forming and decomposing is interesting), under $\rho=120$ a small addition of $R_{s}$ can generate more plugs than under $\rho=50$. The velocity predominance of cars against trucks is declined under the phases of synchronized traffic or wide moving jams. At these phases, flow and mean velocity are determined mainly by vehicle density. Therefore, the range of $R_{s}$ that flow varies over to reach its stationary value under $\rho=120$ is shorter than that under $\rho=50$. We can unchain the slow vehicle entry ban under the phases of synchronized traffic or wide moving jams for that the ban has little effect on flow increasing. And the constraints on slow vehicles can be applied flexibly according to different situations. For example, it is unpractical to keep all slow vehicles out of highways in the region with road network underdeveloped. In this situation, we can make a flexible overtaking ban of slow vehicles to achieve a higher flow and make drivers happy.

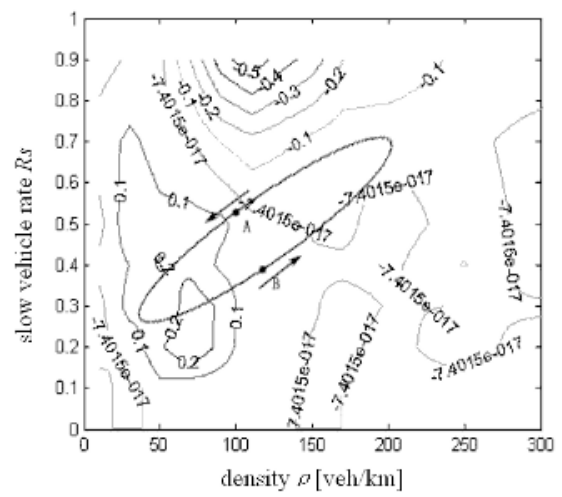

Fig. 4. Contour of $\left(\right.$ Flow $_{\text {forbid }}-$ Flow $\left._{\text {allow }}\right) /$ Flow $_{\text {forbid }}$ as a function of flow density $\rho$ and slow vehicle rate $R_{s}$. Here Flow $_{\text {forbid }}$ and Flow allow $_{\text {represent the traffic flow }}$ when slow vehicles are forbidden to change to the left lane or allowed respectively

Suppose that the ellipse in figure 4 represents density and $R_{s}$ of a two-lane highway as a function of time in one day. The arrow represents the direction that time goes by. The A and B are the moments of day. In order to achieve higher flow, slow vehicles should observe the overtaking ban from moment A to moment B. However, from moment B to moment A the slow vehicle overtaking ban should be cancelled. 


\section{Conclusion}

We redefined the velocity updating rules of NaSch model with the consideration of velocity preference of driver, and extended it with a sophisticated lane changing rule set to an asymmetric two-lane model. The model reproduced empirical results on macroscopic level, i.e. density dependence of lane change frequency and the lane usage inversion. And we found that active lane changes provide more chances for driver to drive at a high and satisfying velocity at free flow phase with the slow vehicle overtaking ban. However, the effect of lane change will vanished without the ban since the vehicle plugs are easier to be formed by structured slow vehicles than to be decomposed only by active lane changes. And even a low fraction of slow vehicle can make the mean velocity and flow go down steeply. In order to weaken the negative impact of vehicle plugs and achieve higher flow, we should keep slow vehicles out of expressway or make a flexible overtaking ban of slow vehicle according to practical situations.

With a simple local interaction set, CA model can reproduce most empirically observed results of complex traffic system and present microscopic causes of formulations of the empirical results. Since the traffic simulations based on CA are at the stage of developing and most analyzes are qualitative. There are many works could be done, for example, clarifying the quantitative relations between simulated and realistic results for quantitative analysis.

\section{References}

[NS] Nagel K., Schreckenberg M.: A cellular automaton model for freeway traffic. Journal de Physique I. 2 (1992) 2221-2229

[SE] Scheider J., Ebersbach A.: Anticipatory drivers in the Nagel-Schreckenberg model. International Journal of Modern Physics C. 13 (2002) 107-113

[KSS1] Knospe W., Santen L., Schadschneider A., Schreckenberg M.:Towards a realistic microscopic description of highway traffic. J. Phys. A. Math. Gen. 33 (2000) 477-485

[KSS2] Knospe W., Santen L., Schadschneider A., Schreckenberg M.. Single-vehicle data of highway traffic: microscopic description of traffic phases . Phys. Rev. E. 65 (2002) 056133

[KSS3] Knospe W., Santen L., Schadschneider A., Schreckenberg M.. A realistic twolane traffic model for highway traffic. J. Phys. A 35 (2002) 3369-3388

[KSS4] Knospe W., Santen L., Schadschneider A., Schreckenberg M.. Disorder effects in cellular automata for two-lane traffic. Physica A. 265 (1999) 614-633

[RN] Rickert M., Nagel K., Schreckenberg M., Latour A.: Two lane traffic simulations using cellular automata. Physica A. 231 (1995): 534-550

[GY] Ganglen C., Yangming K. An empirical investigation of macroscopic lanechanging characteristics on uncongested multilane freeways. Transportation Research Part A. 25 (1991) 375-389

[HL] Hall F. L., Lam T. N.: The characteristics of congested flow on a freeway across lanes, space, and time. Transportation Research Part A. 22 (1988) 45-56 